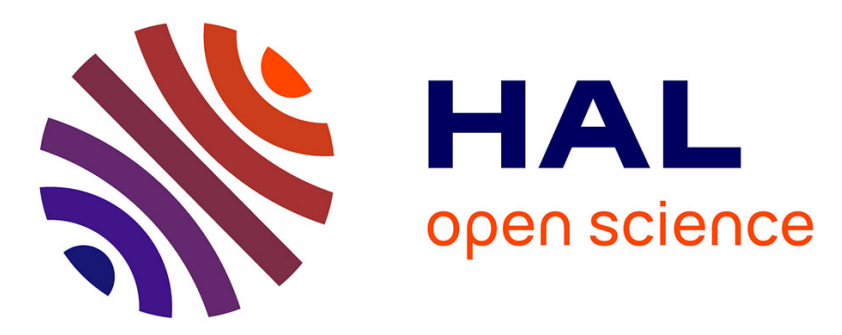

\title{
Dynamic reconfiguration of a compact active stereovision system with digital electromagnetic actuators
}

\author{
Yingfan Hou, Erwan Dupont, Laurent Petit, Tanneguy Redarce, Frédéric
}

\author{
Lamarque
}

\section{To cite this version:}

Yingfan Hou, Erwan Dupont, Laurent Petit, Tanneguy Redarce, Frédéric Lamarque. Dynamic reconfiguration of a compact active stereovision system with digital electromagnetic actuators. 2014 IEEE/ASME International Conference on Advanced Intelligent Mechatronics (AIM), Jul 2014, Besançon, France. pp.1128-1133, 10.1109/AIM.2014.6878232 . hal-02109872

\section{HAL Id: hal-02109872 \\ https://hal.science/hal-02109872}

Submitted on 25 Apr 2019

HAL is a multi-disciplinary open access archive for the deposit and dissemination of scientific research documents, whether they are published or not. The documents may come from teaching and research institutions in France or abroad, or from public or private research centers.
L'archive ouverte pluridisciplinaire HAL, est destinée au dépôt et à la diffusion de documents scientifiques de niveau recherche, publiés ou non, émanant des établissements d'enseignement et de recherche français ou étrangers, des laboratoires publics ou privés. 


\title{
Dynamic reconfiguration of a compact active stereovision system with digital electromagnetic actuators
}

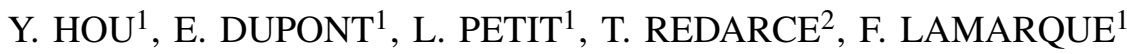

\begin{abstract}
In active-stereovision measurement systems, there is usually only one static configuration for the measuring process. In this paper, a new miniaturized system which can switch dynamically between two active stereovision setups is presented. This system can change its internal configuration in less than $25 \mathrm{~ms}$ and consequently can extract 3D shape information that would not be accessible with only one static configuration. This configuration switch is realized by using two discrete positions digital electromagnetic actuators. The miniaturization is achieved by integrating flexible image guides connected to a compact optical probe. An experimental prototype has been designed, manufactured and tested. Experimental results are presented to demonstrate the feasibility of this new proposed system.
\end{abstract}

\section{INTRODUCTION}

Three dimensional (3D) models reconstruction of object surfaces based on optical measurement is a well-known problem. Many application domains have been explored and studied in recent years, like modeling small objects from images [1], face recognition [2], organs' surface analysis in medical devices [3], 3D road model in vehicle driving assistant [4], etc. The methods to achieve these reconstruction objectives are also various, such as fringe projection [5], stereo matching [1], structure from shading [6], shape from motion [7], etc. However, all these methods have their own pros and cons and it is still a challenging problem to obtain accurate 3D models by using only one of these approaches, especially for irregular shapes and complex surface structures.

Among all these approaches, active stereovision is one of the main methods to achieve 3D reconstruction. Most active stereovision methods are based on two channels to realize the triangulation, one channel for projecting fringe patterns and the other one for acquiring them. The three dimensional depth information of object is obtained through a stereoscopic angle between these two channels. A review of the active methods for stereovision is proposed in [5]. These methods can be applied in cramped environment such as endoscopy [8], [9], or in a miniature device for 3D tooth restoration [10].

Although active stereovision methods can extract surface information with good quality, some factors such as surface

\footnotetext{
${ }^{1}$ Yingfan HOU, Erwan DUPONT, Laurent PETIT and Frédéric LAMARQUE are with Université de Technologie de Compiègne (UTC), Laboratory Roberval(UMR 7337), 1, rue du Dr Schweitzer 60200 Compiègne, France yingfan. houlutc.fr

${ }^{2}$ Tanneguy REDARCE is with Institut National des Sciences Appliquées de Lyon (INSA-Lyon), Laboratoire Ampère(UMR 5005), 20 av Albert Einstein 69100 Villeurbanne, France tanneguy. redarcedinsa-lyon. fr
}

occlusions, shadows, specular areas or absorptive materials can lead to loss of 3D information during the reconstruction process. One basic principle to overcome some of these difficulties is to capture more images of the object in different positions [11], with the drawback of time consumption. Another possibility, proposed in this paper, is to dynamically generate two distinct measurement results by switching between the projection and the acquisition channels. Combining these two results can be an additional way to enrich the surface information obtained from the measurement. In order to achieve miniature system, the flexible image guides combined with compact optical probes have been integrated to assure the miniaturization [12]. To our knowledge, no such system was previously reported in the literature.

In the proposed system, two digital electromagnetic actuators equipped with a mobile mirror are used to change the internal system configuration. Digital actuators have been used because this type of actuators provides a holding force at its discrete states without the need of external electrical energy to hold the positions [13], [14]. Digital actuators are easy to control and can be operated in open loop without the need of sensor integration [13]. In literature, electrostatic [15], electro-thermal [13], piezoelectric [16] or electromagnetic [14], [17] principles have been used for switching the mobile part of the digital actuators between the discrete positions. The electromagnetic principle has been used for the present actuator because a non-consuming holding force can indeed be easily obtained with magnetic interactions between permanent magnets.

The structure of our system and its measurement principle are described in section II. Section III presents the principle of the digital actuators used for the reconfiguration of the measurement system. The experimental setup of the system has been discussed in section IV and measurement results are provided in section V. Finally, the conclusion and perspectives of our work are presented in section VI.

\section{SYSTEM STRUCTURE DESCRIPTION}

The optical and mechanical configurations of the active stereovision systems are generally static. By integrating digital actuators in the active stereovision system, we propose to enhance its $3 \mathrm{D}$ reconstruction capability.

A schematic view of the presented measuring system is shown in Fig.1(a). The projection channel generates fringe patterns by using a DMD (Digital Micro-mirror Device), which is a MEMS device composed of $1024 \times 768$ squared mirrors of $10.8 \mu \mathrm{m}$ pitch and fabricated by Texas Instrument Inc. Each micro-mirror of the DMD can be tilted between 


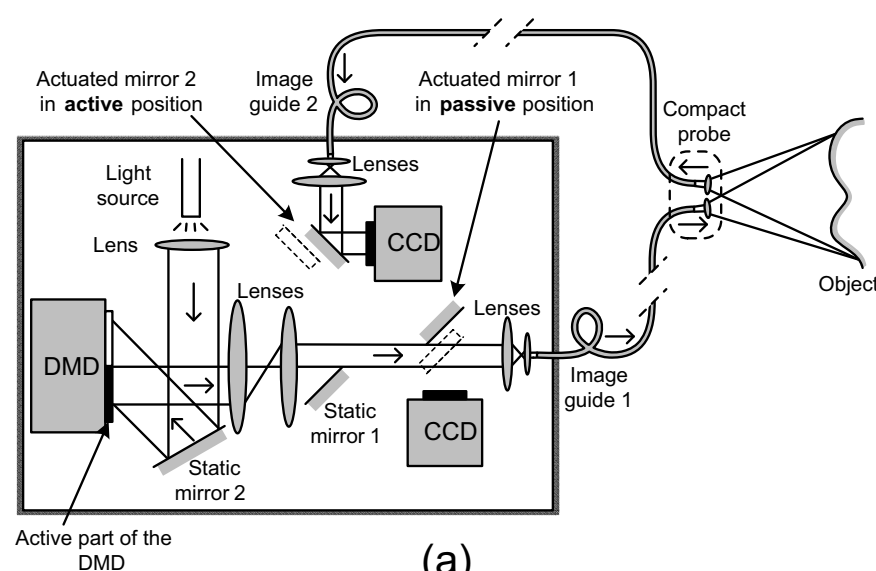

DMD

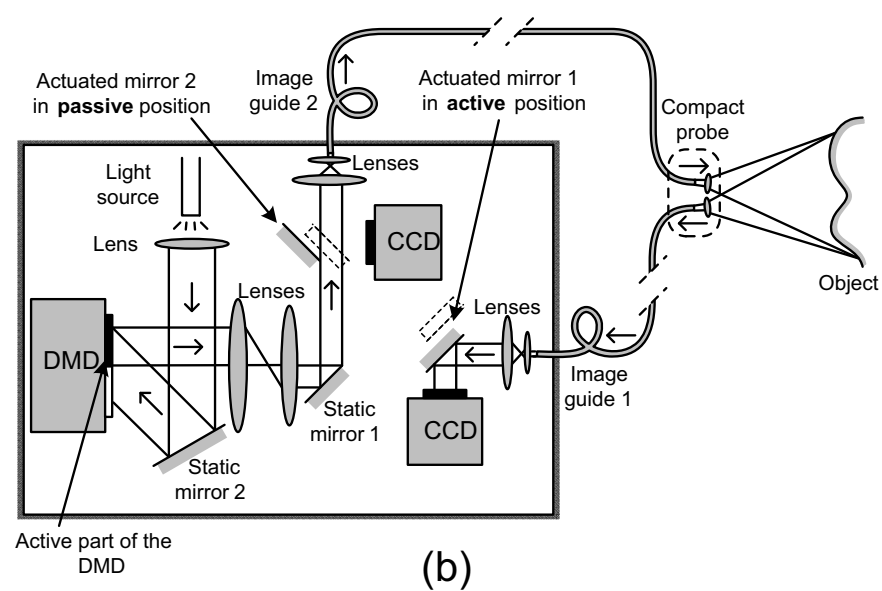

Fig. 1. Principle of the measurement modes.(a): active mode 1.(b): active mode 2 .

two discrete angular positions, and only one of these two positions transmits the light in the following optical interface. A white light source illuminates the DMD, and half of the pattern is finally injected into the image guide 1 . The image guide consists of a fiber bundle composed of 70000 optical fibers with a total diameter of $1.4 \mathrm{~mm}$ over a 2 meters length. It is connected to a miniaturized optical probe composed of a diaphragm and objective lenses as presented in [12].

The pattern coming from the image guide 1 enters into the optical probe and is projected onto the object surface. The light reflected by the object surface is then captured by the miniaturized optical probe and injected into the image guide 2. Then the image of the measure area is finally focused on a CCD sensor composed of $1024 \times 768$ pixels. The analysis of the deformed pattern on the captured image will finally give the depth information of the measured surface. As shown in Fig.1(a), three mirrors are used: static mirror 2 and two actuated mirrors. Static mirror 2 is used to reflect the light onto DMD from a light source. The actuated mirror 1 is in passive position, which builds an active channel. One half of the DMD is used to inject a pattern in the image guide 1, because only half of the DMD is active to reflect the light although the entire DMD is covered by light, as shown in Fig.1(a). In addition, the fringe patterns acquisition channel is realized by setting the actuated mirror 2 in active position to reflect the image coming from the image guide 2 into a CCD sensor.

By switching the position of both actuated mirrors using digital actuators, another active stereovision configuration is obtained as represented in Fig.1(b). In this configuration, the projection and acquisition channels are reversed with a help of the fourth mirror (i.e., static mirror 1). Another half of the DMD is active to inject a pattern onto the image guide 2 , with a reflection on the static mirror 1 . A second CCD sensor is used to get the image from the image guide 1.In the following, the configuration of Fig.1(a) will be called "mode 1" and Fig.1(b) "mode 2".

Because the projection and acquisition channels are exchanged between mode 1 and mode 2 , the captured images are different in these two modes although the object and measure area are the same. In fact, two different view angles are realized in mode 1 and mode 2, different sides of the objects can be seen by the capture channel and the measurement surface information will be enriched to reconstruct a more precise 3D model.

\section{DIGITAL ACTUATOR PRINCIPLE}

To move the actuated mirrors, two identical digital actuators providing two discrete positions have been integrated. A schematic layout of the digital actuator working principle is provided in Fig.2. The actuator consists of a Mobile Permanent Magnet (MPM) placed in a rectangular cavity, two Fixed Permanent Magnets (FPMs) and five current carrying wires

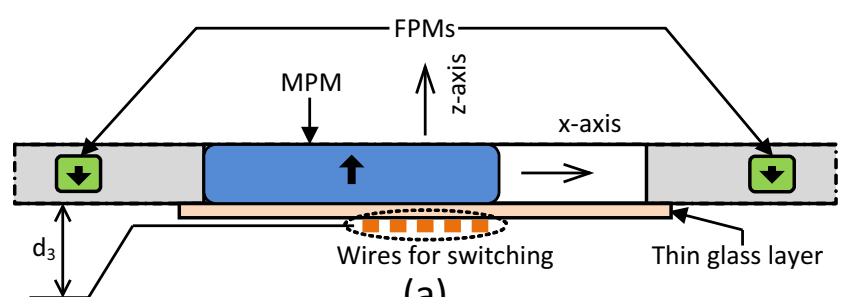

(a)

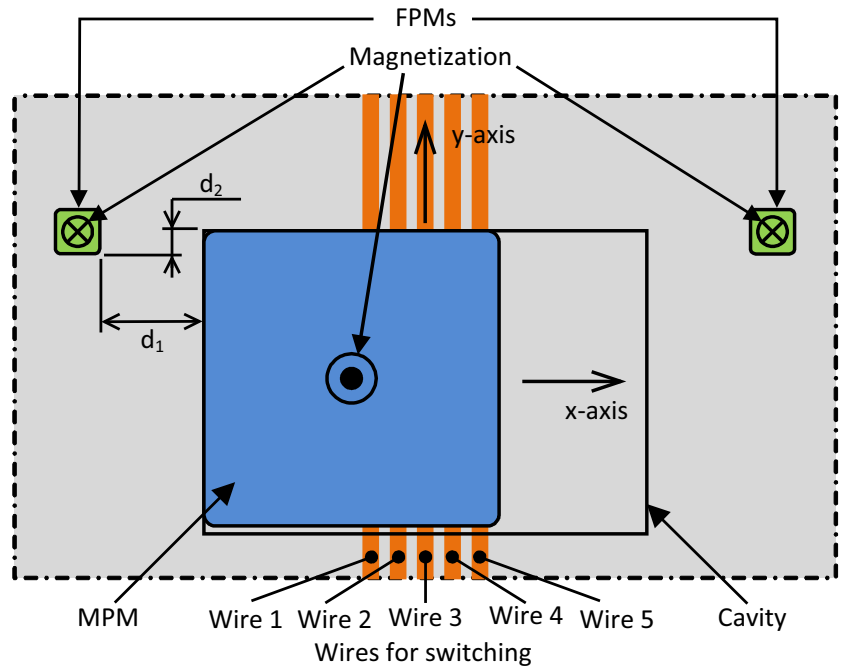

(b)

Fig. 2. Schematic layout of the digital actuator.(a): Side view.(b): Top view 
placed at the center of the cavity. The MPM can only move along the $\mathrm{x}$-axis and reach two discrete positions located at the extremities of the stroke. At the discrete position, the FPMs exert a magnetic holding force on the MPM which eliminates the need of electrical energy to maintain the discrete positions even in presence of external disturbances (e.g., vibrations, etc). The switching of the MPM between the discrete positions is obtained electromagnetically, i.e., generating a Lorentz force by injecting the current inside the current carrying wires placed below the cavity. The actuator stroke is $5 \mathrm{~mm}$ and has been defined according to the optical beam diametre (coming from the DMD or the image guides, see Fig.1). The digital actuator characteristics are provided in Table I.

The magnetic holding force exerted by the FPMs on the MPM has two components along $\mathrm{x}$ and $\mathrm{y}$-axes whose values are $22.1 \mathrm{mN}$ and $11.9 \mathrm{mN}$ respectively. The objective of these components is to ensure a high positioning repeatability of the MPM at its discrete positions along the two axes. The holding force value along the $\mathrm{x}$-axis has been determined by considering the adhesion effect and the mass of the actuated mirror. With this holding force value, if the mobile part (MPM + actuated mirror) is placed at a distance of $1.38 \mathrm{~mm}$ from its discrete position, it will reach the discrete position without electrical energy consumption (magnetic attraction). Because of assembly constraints, the dimension of the cavity along the $y$-axis is slightly higher $(0.1 \mathrm{~mm})$ than the MPM dimension. The $y$-axis holding force has been implemented in order to ensure the contact between the MPM and the lateral stop during the switch.

In order to obtain a high driving force and a $5 \mathrm{~mm}$ stroke actuator, five electrical wires are used and connected in series. A distance of $250 \mu \mathrm{m}$ between two wires has been used to maximize the electromagnetic generated force. The wire

TABLE I

DIGITAL ACTUATOR CHARACTERSISTICS

\begin{tabular}{|c|c|c|}
\hline \multicolumn{3}{|c|}{ Materials } \\
\hline \multirow{2}{*}{\multicolumn{2}{|c|}{$\begin{array}{l}\text { Permanent magnets } \\
\text { Mechanical structure }\end{array}$}} & Neodium-Iron-Boron (NdFeB) \\
\hline & & VeroWhite-FullCure® 830 \\
\hline Permanent magnets & Dimensions $(\mathrm{mm})$ & $\overline{\text { Remnant Magnetizations(T) }}$ \\
\hline MPM & $10 \times 10 \times 2$ & 1.35 \\
\hline FPM & $1.5 \times 1.5 \times 1.2$ & 1.17 \\
\hline \multicolumn{3}{|c|}{ Distances(Fig.2(b)) } \\
\hline Stro & & $5 \mathrm{~mm}$ \\
\hline$d_{1}$ & & $3.5 \mathrm{~mm}$ \\
\hline$d_{2}$ & & $1.15 \mathrm{~mm}$ \\
\hline$d_{3}$ & & $222 \mu \mathrm{m}$ \\
\hline \multicolumn{3}{|c|}{ Forces } \\
\hline Magnetic holdin & orce $(x$-axis $)$ & $22.1 \mathrm{mN}$ \\
\hline Magnetic holdin & force (y-axis) & $11.9 \mathrm{mN}$ \\
\hline Electromagnetic dri & ig force $(x$-axis $)$ & $9.5 \mathrm{mN}$ for $1 \mathrm{~A}$ \\
\hline \multicolumn{3}{|c|}{ Masses } \\
\hline MP & & $1.50 \mathrm{~g}$ \\
\hline Mir & & $0.16 \mathrm{~g}$ \\
\hline \multicolumn{3}{|c|}{ Adhesion coefficients } \\
\hline MPM - Gl & s layer & 0.41 \\
\hline MPM - & $\mathrm{BS}$ & 0.27 \\
\hline
\end{tabular}

width and thickness are $500 \mu \mathrm{m}$ and $35 \mu \mathrm{m}$, respectively. A modeling of the magnetic and electromagnetic forces exerted on the MPM has been carried out in RADIA software. The Fig.3(a) represents the electromagnetic force exerted on the MPM with a $1 A$ driving current. The electromagnetic force exerted by each wire and the total force exerted by the five wires are represented in Fig.3(a) in function of the MPM position. Because of the variation of the relative position between the MPM and the wires, the electromagnetic force generated by each wire is different and non-symmetrical according to the cavity center (i.e., $0 \mathrm{~mm}$ ). When the MPM is placed at the left position (i.e., $-2.5 \mathrm{~mm}$ ), the maximum and minimum electromagnetic forces generated by Wire 1 and Wire 5 are found to be $2.4 m N$ and $1.5 m N$, respectivelly. Similar forces are generated when the MPM is placed at the right position (i.e., $+2.5 \mathrm{~mm}$ ). The total electromagnetic force generated with the five wires is $9.5 \mathrm{mN}$ when the MPM is located in a discrete position (i.e., $\pm 2.5 \mathrm{~mm}$ ). A vertical force (along z-axis) is also exerted on the MPM when it is misaligned with respect to the center of the cavity. The maximum value of this vertical force is $\pm 3.7 \mathrm{mN}$ when MPM is at its disctete position.

The Fig.3(b) represents the total force (magnetic + electromagnetic) exerted on the MPM in function of its position in the cavity for three driving current values. In the first configuration (Driving current $=0 A$ ), only the magnetic holding force exerted on the MPM is visible. When the

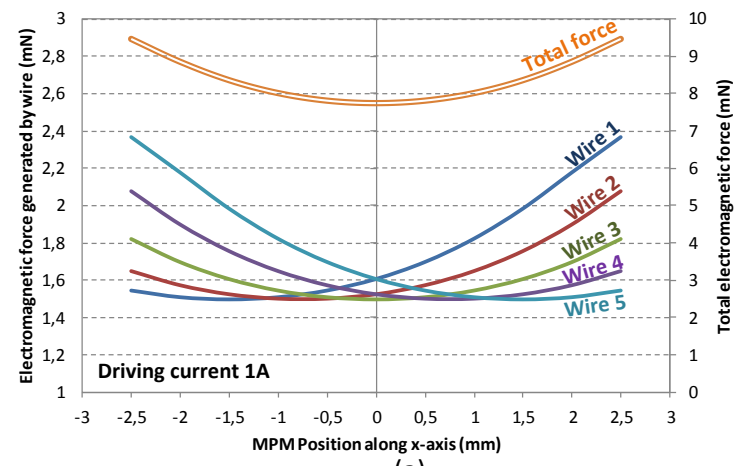

(a)

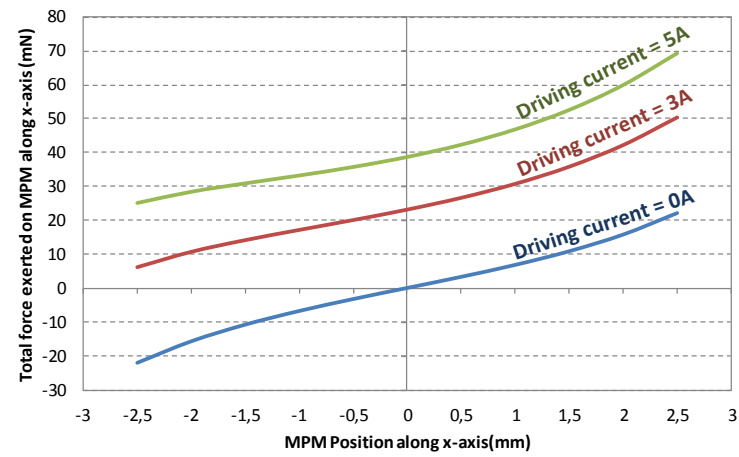

(b)

Fig. 3. Simulated results.(a):Electromagnetic force generated by the five wires $(1 \mathrm{~A}$ driving current).(b):Total force exerted on MPM in function of its position. 
MPM is located in discrete position, a holding force of $\pm 22.1 \mathrm{mN}$ is visible. When a driving current is added, the initial curve (Driving current $=0 A$ ) is vertically shifted. Two configurations are represented in Fig.3(b) with $3 A$ and $5 A$. Considering the magnetic holding and the adhesion forces, the minimum driving current necessary to switch the mobile part (MPM + mirror) is found to be 3.38A .

A dynamic model of the actuator has been developped and is described in [18]. Using this model, the MPM displacement in function of time has been determined and is presented in Fig.4. Driving current pulses with 5A amplitude and with different widths have been considered. With a pulse width of $8 m s$, the MPM does not switch because the pulse width is too small. In this configuration, the MPM returns to the initial position because of the magnetic holding force exerted by the FPMs. With pulse width of $11 \mathrm{~ms}$, the MPM stops in the position close to the central position. This configuration is not acceptable for the digital actuators because in a normal functionning, the MPM can only stop in one of the discrete positions. With a pulse width of $30 \mathrm{~ms}$, the MPM reaches the second stable position before the end of the pulse. The minimal pulse width is then obtained in this configuration. In this configuration, the optimal switch time is $24.4 m s$.

\section{EXPERIMENTAL REALIZATION}

An experimental prototype of the measurement system which integrates two digital actuators has been manufactured and is presented in Fig.5. Fig.5(a) represents the measurement probe and the measure area. Two image guides (FIGH60-1200N, FUJIKURA) are combined in a compact probe with lenses and diaphragms. The probe size dimensions are $55(\mathrm{~L}) \times 18(\mathrm{~W}) \times 10(\mathrm{H})(\mathrm{mm})$. Objects to be measured are placed in front of the probe. The stereovision angle between the two image guides is realized by the mirror placed next to the measure area. By shifting the image guide through the focusing stages, an optical conjugation between the object to be measured and the ends of the image guides can be obtained. More details regarding this procedure is provided in [12].

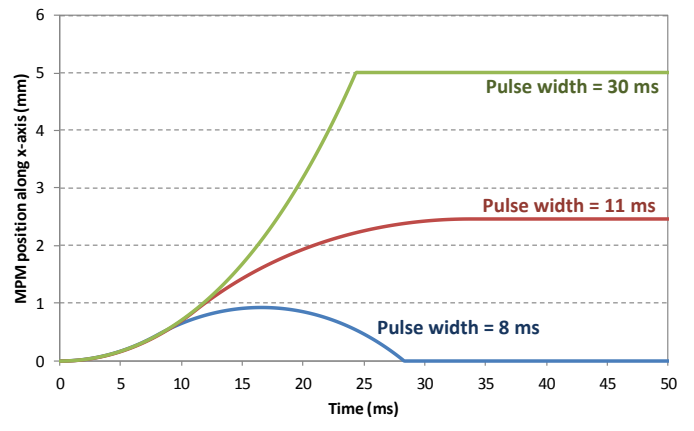

Fig. 4. Simulated MPM displacement with $5 A$ driving current and for different pulse widths
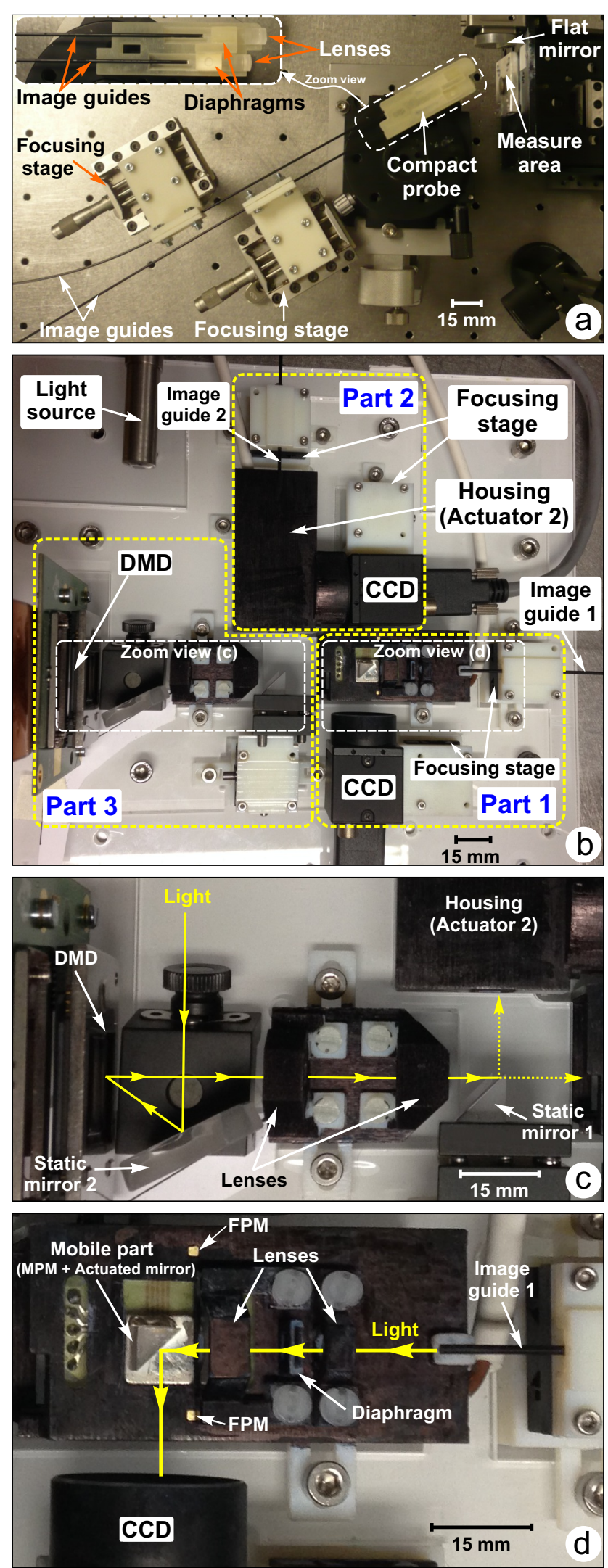

Fig. 5. Photography of the 3D measurement system.(a):measurement part.(b):controle part.(c):enhanced image of Part 3 in (b).(d):enhanced image of Part 1 in (b). 
The active part of the measuring system consists of three parts as shown as in Fig.5(b). Part 1 and Part 2 are identical; they contain cameras, image guides, mechanical supports for actuators and lenses, and a digital actuator, which moves an actuated mirror (see Fig.5(d)). Two cameras (Flea ${ }^{\circledR} 2$ CCD Camera from Point Grey Inc.) capture the images reflected by the actuated mirrors. The cameras and image guides are supported by translation stages for focusing purposes. In order to reduce the noise on the CCD sensors, black housings are added in the top side of the digital actuators. In Part 2, the housing is visible but not in Part 1 because this one has been removed in order to see the digital actuator. They prevent useless external light from entering into the optical system and into the cameras. The mechanical supports are covered by the black housings in Part 1 and Part 2 .

Fig.5(c) is an enhanced image of Part 3, which represents the light travel before and after the DMD reflection. Part 3 is composed of a static mirror, two imaging lenses on a mechanical support and the DMD. A light source and a mirror are used to light up the DMD. The yellow line is the light path and indicates the angle of incident light on the DMD. This angle should be accurately controlled, because the quality of generated patterns is very sensitive to its value when the patterns are projected into Part 1 or Part 2.

Fig.5(d) represents an enhanced image of Part 1, which shows the digital actuators integrated in the design of the measurement system. The figure shows the mechanical supports, optical elements and actuators both used in Part 1 and Part 2 (which are identical). The yellow line indicates the light path for the case of acquisition by CCD. The actuator support has been manufactured using 3D printing machine by STRATASYS Ltd, and the materials used are VeroWhite - FullCure® 830, which are from Objet Geometries Ltd. The control of the two digital actuators is realized using a computer equipped with a data acquisition board NI 6289 (National Instrument) and a voltage to current converter (linear conversion, sampling rate of $50 \mathrm{kHz}$, input $[-10 \mathrm{~V}$; $+10 \mathrm{~V}]$ and output $[-10 A ;+10 A])$.

The three parts of the whole system are supported by two $5 \mathrm{~mm}$ thick white plastic boards, each having dimensions $305 \mathrm{~mm} \times 245 \mathrm{~mm}$, which are fixed on a marble table.

\section{MEASUREMENT RESULTS}

Experimentations have been carried out with the presented prototype and the two active modes have been tested. A 10 cent euro coin has been used as measured object as shown in Fig.6. The letter "N" of the "CENT" word has been measured and the results are presented in Fig.7.

For the active mode 1 and mode 2, vertical line patterns are projected on the object by one channel and then captured by the camera with the other one. Fig.7(a) and Fig.7(b) show the capture results for the two modes, respectively. Vertical lines of the projected patterns are deformed because of the height of letter $\mathrm{N}$. The orientation of patterns deformation are opposite between Fig.7(a), patterns deformed to the left, and Fig.7(b), patterns deformed to the right. This can also be seen in Fig.7(c) and Fig.7(d), which are the analyzed

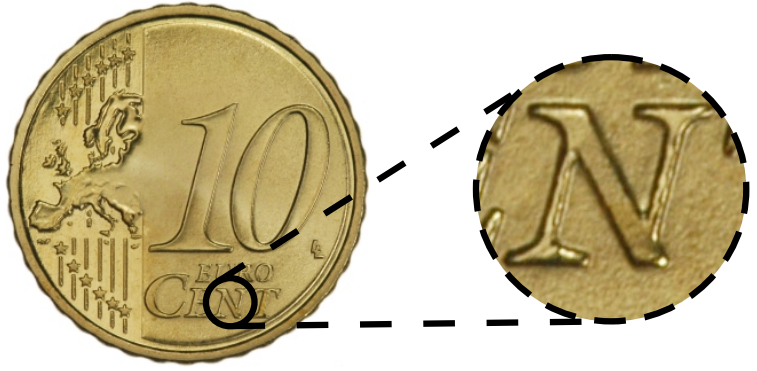

Fig. 6. The letter $\mathrm{N}$ is measured from a 10 cent of euro coin.

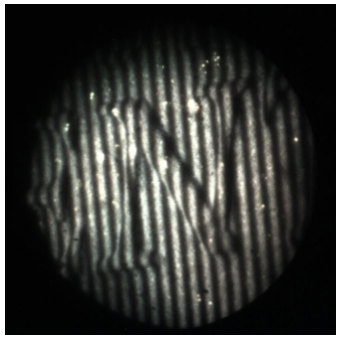

(a)

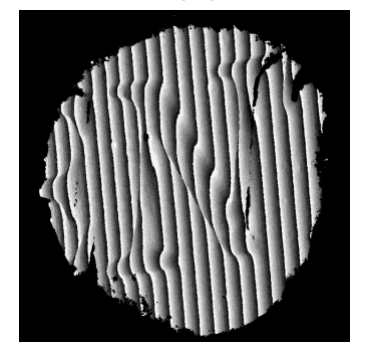

(c)

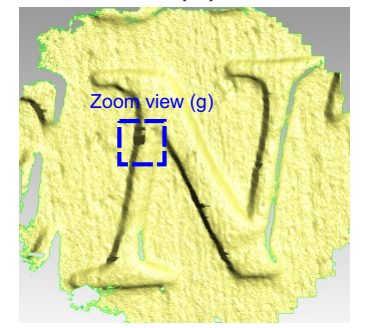

(e)

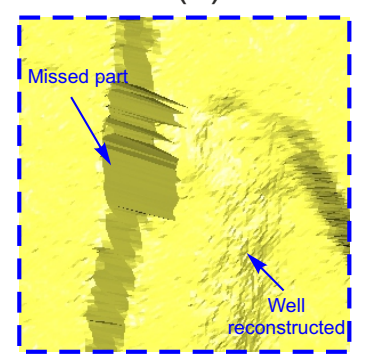

(g)

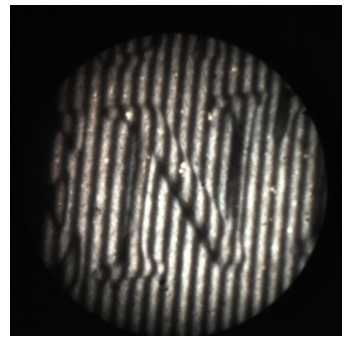

(b)

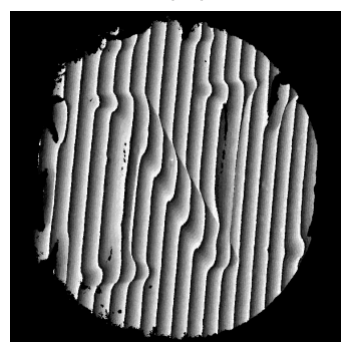

(d)

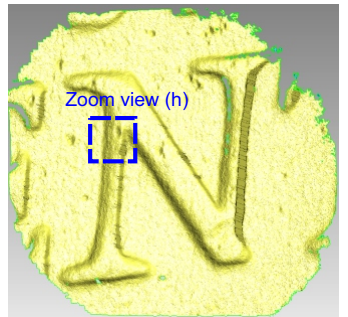

(f)

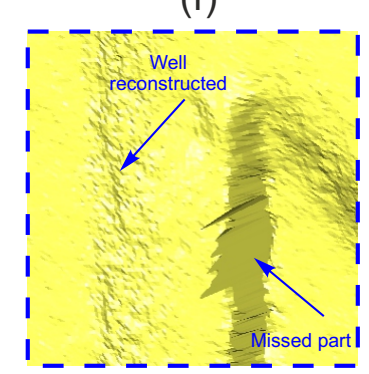

(h)
Fig. 7. Experimental measurement results. (a) and (b): result of active mode 1 and 2. (c) and (d): image of analyzed phase for mode 1 and 2. (e) and (f): reconstruction results of mode 1 and mode 2. (g) and (h): enhanced image for (e) and (f).

phase of Fig.7(a) and Fig.7(b). The opposite orientation of patterns' deformation indicates that active mode 1 and mode 
2 measure the same object from two different points of view. To reconstruct the 3D model, these two different sides of measurement will generate two 3D surface models as shown in Fig.7(e) and (f), which are generated by applying the methods mentioned in [12]. In Fig.7(e), some parts of the letter $\mathrm{N}$ have not been well reconstructed (e.g. the missed part of lettre $\mathrm{N}$ in Fig.7(g)) but they are well reconstructed in Fig.7(f) (e.g. the well reconstructed part of letter $\mathrm{N}$ in Fig.7(h)) and vice versa. Using the proposed system, the final 3D reconstruction can then be enriched by combining the 3D models obtained from the two modes, that will be studied in future work. The presented experimental results show that a dynamic reconfiguration of the measuring system has been developed using two digital actuators in order to obtain two active modes. The results point toward the potential gains from combining these two modes for better 3D reconstruction.

\section{CONCLUSIONS}

In this paper, a new miniaturized measuring system able to be dynamically reconfigured between two active stereovision modes to measure the $3 \mathrm{D}$ shape of objects is presented. In less than $25 \mathrm{~ms}$, the projection and the acquisition channels can be exchanged to obtain complementary 3D measurements of the same object. The dynamic system reconfiguration is based on two identical digital electromagnetic actuators having two discrete positions. A prototype of the measuring system has been manufactured and its feasibility has been validated. Experimental results have been presented for the two modes and the benefit of the reconfiguration has been clearly observed.

In future works, a new version of this stereovision system will be developed to enhance the projection channel resolution by using the full DMD surface instead of half DMD surface for each active mode. It is also planned to miniaturize the size of the system, especially for actuators, to reduce the switching time of actuators and to realize the measurement in high frequency. Some more complex objects will be tested to verify the performance of this measurement system.

\section{REFERENCES}

[1] W. K. Leow, Z. Huang, Y. Zhang, and R. Setiono, "Rapid 3d model acquisition from images of small objects," in Geometric Modeling and Processing 2000. Theory and Applications. Proceedings. IEEE, 2000, pp. 33-41.

[2] A. Aissaoui, J. Martinet, and C. Djeraba, "3d face reconstruction in a binocular passive stereoscopic system using face properties," in Image Processing (ICIP), 2012 19th IEEE International Conference on. IEEE, 2012, pp. 1789-1792.
[3] X. Maurice, C. Albitar, C. Doignon, and M. de Mathelin, "A structured light-based laparoscope with real-time organs' surface reconstruction for minimally invasive surgery," in Engineering in Medicine and Biology Society (EMBC), 2012 Annual International Conference of the IEEE. IEEE, 2012, pp. 5769-5772.

[4] J.-P. Tarel, P. Charbonnier, F. Goulette, and J.-E. Deschaud, "3d road environment modeling applied to visibility mapping: an experimental comparison," in Proceedings of the 2012 IEEE/ACM 16th International Symposium on Distributed Simulation and Real Time Applications. IEEE Computer Society, 2012, pp. 19-26.

[5] S. S. Gorthi and P. Rastogi, "Fringe projection techniques: whither we are?" Optics and lasers in engineering, vol. 48, no. 2, pp. 133-140, 2010.

[6] E. Prados and O. Faugeras, "Shape from shading," in Handbook of mathematical models in computer vision. Springer, 2006, pp. 375388.

[7] T. Collins, B. Compte, and A. Bartoli, "Deformable shape-frommotion in laparoscopy using a rigid sliding window," in Medical Image Understanding and Analysis Conference, 2011.

[8] C. Wong, N. Chen, and C. Sheppard, "Study on potential of structured illumination microscopy utilizing digital micromirror device for endoscopy purpose," in Biophotonics, Nanophotonics and Metamaterials, 2006. Metamaterials 2006. International Symposium on. IEEE, 2006, pp. 218-221.

[9] E. Dupont, F. Lamarque, C. Prelle, T. Redarce, et al., "Tri-dimensional optical inspection based on flexible image guide: first step toward $3 \mathrm{~d}$ industrial endoscopy," in Proc. of the 11th Biennial Conference on Engineering Systems Design and Analysis, vol. 19, 2012.

[10] H. Cui, N. Dai, W. Liao, and X. Cheng, "Intraoral 3d optical measurement system for tooth restoration," Optik-International Journal for Light and Electron Optics, vol. 124, no. 12, pp. 1142-1147, 2013.

[11] H. Cui, W. Liao, N. Dai, and X. Cheng, "Registration and integration algorithm in structured light three-dimensional scanning based on scale-invariant feature matching of multi-source images," Chinese Optics Letters, vol. 10, no. 9, pp. 091 001-091 001, 2012.

[12] E. Dupont, Y. Hou, F. Lamarque, and T. Redarce, "Binary pattern codification strategies in an active stereoscopic system based on flexible image guides," in SPIE MOEMS-MEMS. International Society for Optics and Photonics, 2013, pp. $86180 \mathrm{H}-86180 \mathrm{H}$.

[13] V. Chalvet, Y. Haddab, and P. Lutz, "A microfabricated planar digital microrobot for precise positioning based on bistable modules," 2013.

[14] X. Miao, X. Dai, P. Wang, Y. Huang, G. Ding, and X. Zhao, "Electromagnetic bistable microactuator fabricated on a single wafer," Micro \& Nano Letters, IET, vol. 7, no. 2, pp. 99-100, 2012.

[15] Y. Gerson, S. Krylov, B. Ilic, and D. Schreiber, "Design considerations of a large-displacement multistable micro actuator with serially connected bistable elements," Finite Elements in Analysis and Design, vol. 49, no. 1, pp. 58-69, 2012.

[16] P. F. Giddings, H. A. Kim, A. I. Salo, and C. R. Bowen, "Modelling of piezoelectrically actuated bistable composites," Materials Letters, vol. 65 , no. 9, pp. 1261-1263, 2011.

[17] L. Petit, A. Hassine, J. Terrien, F. Lamarque, and C. Prelle, "Development of a control module for a digital electromagnetic actuators array," 2013.

[18] L. Petit, C. Prelle, E. Doré, F. Lamarque, and M. Bigerelle, "A fourdiscrete-position electromagnetic actuator: modeling and experimentation," Mechatronics, IEEE/ASME Transactions on, vol. 15, no. 1, pp. 88-96, 2010. 